\title{
BMJ Open Comparative efficacy and acceptability of antidepressant treatment in poststroke depression: a multiple- treatments meta-analysis
}

\author{
Yefei Sun, ${ }^{1}$ Yifan Liang, ${ }^{2}$ Yang Jiao, ${ }^{2}$ Jueying Lin, ${ }^{3}$ Huiling Qu, ${ }^{4}$ Junjie $\mathrm{Xu},{ }^{5}$ \\ Chuansheng Zhao ${ }^{2}$
}

To cite: Sun Y, Liang $Y$, Jiao Y, et al. Comparative efficacy and acceptability of antidepressant treatment in poststroke depression: a multiple-treatments meta-analysis. BMJ Open 2017;7:e016499. doi:10.1136/ bmjopen-2017-016499

\section{- Prepublication history and} additional material for this paper are available online. To view these files please visit the journal online (http://dx.doi. org/10.1136/bmjopen-2017016499).

Received 18 February 2017 Revised 18 May 2017 Accepted 5 June 2017

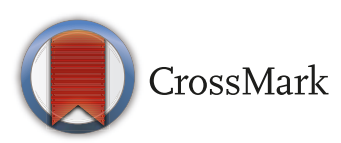

${ }^{1}$ Department of Gastrointestinal Surgery, The First Hospital of China Medical University, Shenyang, China

2Department of Neurology, The First Hospital of China Medical University, Shenyang, China ${ }^{3}$ Department of Emergency, Zhongshan Hospital Xiamen University, Xiamen, China ${ }^{4}$ Department of Neurology, The People's Hospital of Liaoning Province, Shenyang, China ${ }^{5}$ Department of Laboratory Medicine, The First Hospital of China Medical University, Shenyang, China

Correspondence to Yefei Sun; yfsun@cmu.edu.cn

\section{ABSTRACT}

Objective The aim of this study is to create a rank order of the comparative efficacy and acceptability (risk of all-cause discontinuation) of antidepressant treatment in poststroke depression (PSD) by integrating direct and indirect evidence.

Design Multiple-treatments meta-analysis of randomised controlled trials.

Participants Patients with depression following stroke. Interventions 10 antidepressants and placebo in the acute treatment of PSD.

Outcome measures The primary outcomes were the overall efficacy, defined as the mean change of the total depression score. The secondary outcome was the acceptability, defined as risk of all-cause discontinuation. These estimates as standardised mean differences or ORs with $95 \%$ Cls.

Results We identified 12 suitable trials, with data from 707 participants. All drugs were significantly more effective than placebo apart from sertraline, nefiracetam and fluoxetine. Most of the comparisons for acceptability revealed no significant differences except that paroxetine had significantly lower all-cause discontinuation than doxepin, citalopram and fluoxetine. Standardised mean differences compared with placebo for efficacy varied from -6.54 for the best drug (reboxetine) to 0.51 for the worst drug (nefiracetam). ORs compared with placebo for acceptability ranged from 0.09 for the best drug (paroxetine) to 3.42 for the worst drug (citalopram). For the efficacy rank, reboxetine, paroxetine, doxepin and duloxetine were among the most efficacious treatments, the cumulative probabilities of which were $100 \%$, $85.7 \%, 83.2 \%, 62.4 \%$, respectively. With respect to the acceptability rank, paroxetine, placebo, sertraline and nortriptyline were among the most acceptable treatments, the cumulative probabilities of which were $92.4 \%, 63.5 \%$, $57.3 \%, 56.3 \%$.

Conclusion After weighing the efficacy and acceptability, we conclude that paroxetine might be the best choice when starting acute treatment for PSD, and fluoxetine might be the worst choice.

Trial registration number This systematic review has been registered in the Prospective Register of Systematic Review Protocols (PROSPERO) public database (CRD42017054741; http://www.crd.york.ac.uk/ PROSPERO).
Strengths and limitations of this study

- In the absence of head-to-head comparisons, it provided us with a means of ranking different antidepressants against each other for poststroke depression (PSD).

- In the case of limited sample sizes, it strengthened inferences by integrating data from direct and indirect comparisons.

- The study included most of the antidepressants that are used in the clinical treatment of PSD.

- The included randomised controlled trials had small sample sizes and the number of studies on the same kind of antidepressant was too small.

- The trials were for the treatment duration of 6-12 weeks.

\section{INTRODUCTION}

Poststroke depression (PSD) is common, affecting approximately one-third of stroke survivors. ${ }^{1}$ There is abundant evidence indicating that PSD is associated with increased mortality and poor functional outcomes. ${ }^{2-5}$ Although evidence has emerged from systematic reviews to indicate that there are both validated depression screening tools ${ }^{6}$ and effective treatment and prevention strategies for depression after stroke, ${ }^{7-9}$ there has not been any significant reduction in the pooled frequency estimate of patients experiencing PSD (ie, values were $33 \%$ in $2005^{10}$ and $31 \%$ in $2014^{11}$ ). One reason is the high rates of refusal by stroke clinicians to recommend antidepressant therapy, because they consider the therapeutic efficacy of antidepressants for PSD treatment to be insignificant and also being associated with a significant risk of adverse events. ${ }^{12}$ Moreover, currently there are more than 40 different antidepressants in clinical use, which are divided into nine categories. Stroke clinicians seem to have difficulties in making a rational choice about 


\section{PRISMA 2009 Flow Diagram}
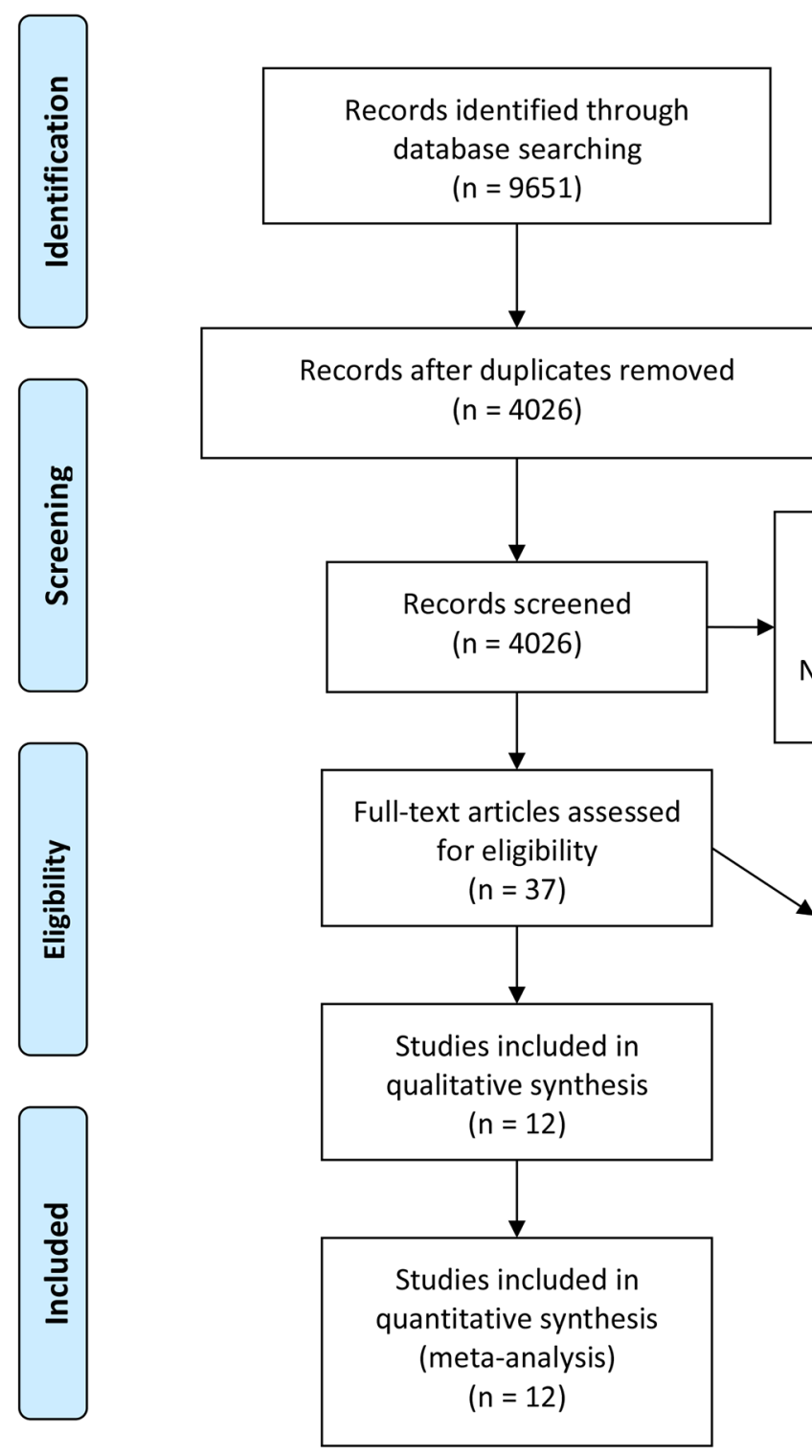

Records excluded

( $n=3989$ )

No relation to PSD $(n=3141)$

No relation to the antidepressant treatment for PSD $(n=800)$

Meta-analysis and review article $(n=48)$

Full-text articles excluded, with reasons

$$
(n=25)
$$

Not a monotherapy $(n=3)$

Cohort study and case report $(n=12)$

Studied without a control group $(n=4)$

Participants are not all PSD $(n=2)$

Data not in our sphere of interest or incomplete $(n=4)$

Figure 1 PRISMA flow diagram. PRISMA, Preferred Reporting Items for Systematic Reviews and Meta-Analyses; PSD, poststroke depression. Reproduced with permission from Moher D, Liberati A, Tetzlaff J, Altman DG, The PRISMA Group (2009) Preferred Reporting Items for Systematic Reviews and Meta-Analyses: The PRISMA Statement. PLoS Med 6(7): e1000097.

which antidepressant to prescribe. Neither the absolute nor the relative efficacy of antidepressants has been fully established. There are even no recommendations in the guideline if any of these different drug classes of antidepressants is superior to the others. ${ }^{13}$ Therefore, whether and what antidepressant treatment for PSD should be prescribed remains controversial. Previous conventional pairwise meta-analyses have not been able to generate clear rank orders for the efficacy and acceptability of available treatments, because many antidepressants have not been compared in a head-to-head manner. ${ }^{7}$ In addition, the number of included randomised controlled trials (RCTs) is limited, which can introduce some bias into any conclusions. Thus, it would be beneficial to create a rank order taking both efficacy and unwanted effects into consideration.

Multiple-treatments meta-analysis is also known as mixed-treatment comparisons meta-analysis or network meta-analysis. ${ }^{14}$ It can provide us with a way to rank different interventions against each other. It also helps 


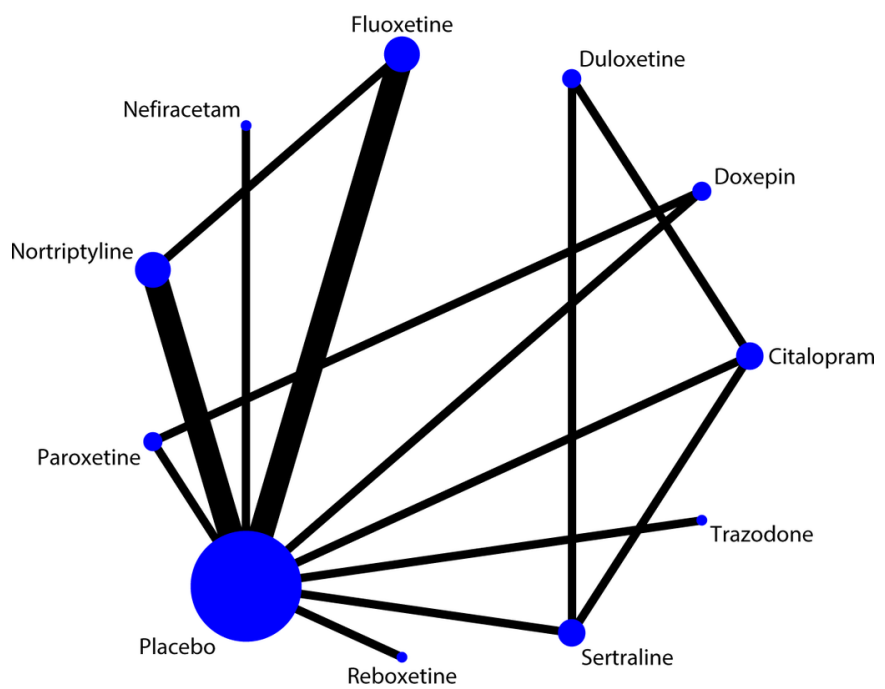

Figure 2 Network plot of the included studies for the multiple-treatments meta-analysis for efficacy. The width of the lines is proportional to the number of studies comparing each pair of treatments, and the size of each node is proportional to the number of randomised participants (sample size). The network plot of included studies for acceptability analysis is similar.

to strengthen inferences of how large the differences are between all the available interventions, since this approach integrates data from direct (when treatments are compared within a randomised trial) and indirect comparisons (when treatments are compared between trials). ${ }^{15}$ We aimed to compare the efficacy and acceptability of antidepressant treatment in PSD by conducting a multiple-treatments meta-analysis. It was intended to create the rank orders of different drugs to alleviate depression while taking into account the risk of all-cause discontinuation. Simultaneously, it would strengthen inferences concerning the relative efficacy and acceptability of antidepressant treatment in PSD.

\section{METHODS}

\section{Criteria for considering studies}

This systematic review has been registered in the Prospective Register of Systematic Review Protocols (PROSPERO) public database (CRD42017054741; http://www.crd. york.ac.uk/PROSPERO).

We included only RCTs that compared antidepressants as monotherapy in the acute-phase treatment of patients with PSD.

The patients with stroke had to be diagnosed clinically and/or by CT scan or MRI. They had also received a diagnosis of depression following stroke, as confirmed by either Diagnostic and Statistical Manual of Mental Disorders (DSM) criteria or some other validated rating scale for depression. There were no inclusion restrictions on the basis of patient and study characteristics, such as age, sex and classes of antidepressants.

We also included placebo in the comparison group, because there is no consensus about either the efficacy or acceptability of antidepressant therapy.

\section{Search methods and study selection}

We searched Medline, Embase, PsycINFO, Cochrane Central Register of Controlled Trials, Web of Science (science and social science citation index) prior to December 2016. Further relevant trials were obtained by manual search of reference lists of all available records identified in the initial search. The authors were contacted for further information regarding unpublished trials and reports found in published databases. Keywords used in the searches were 'depress* AND stroke' (see online supplementary file 1). Various combinations of the search

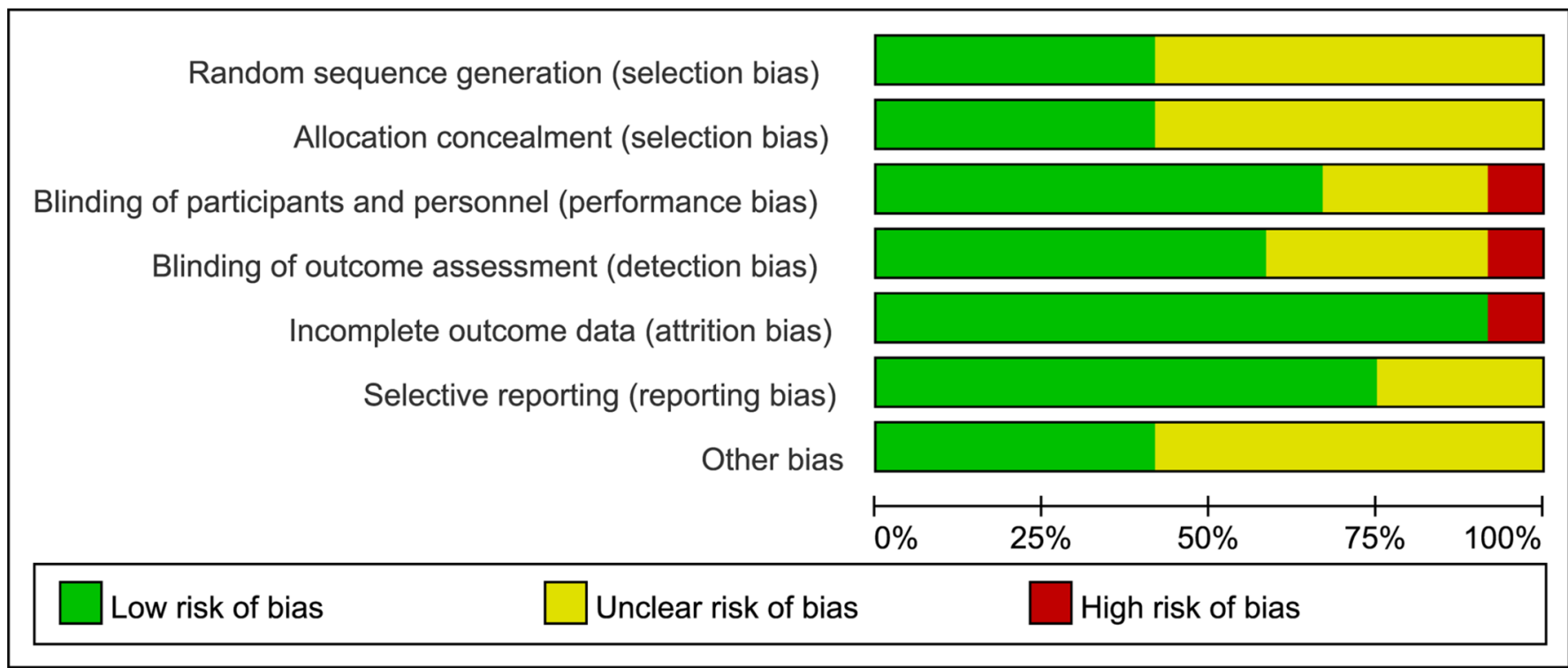

Figure 3 Risk of bias graph: review authors' judgements about each risk of bias item presented as percentages across all included studies. 


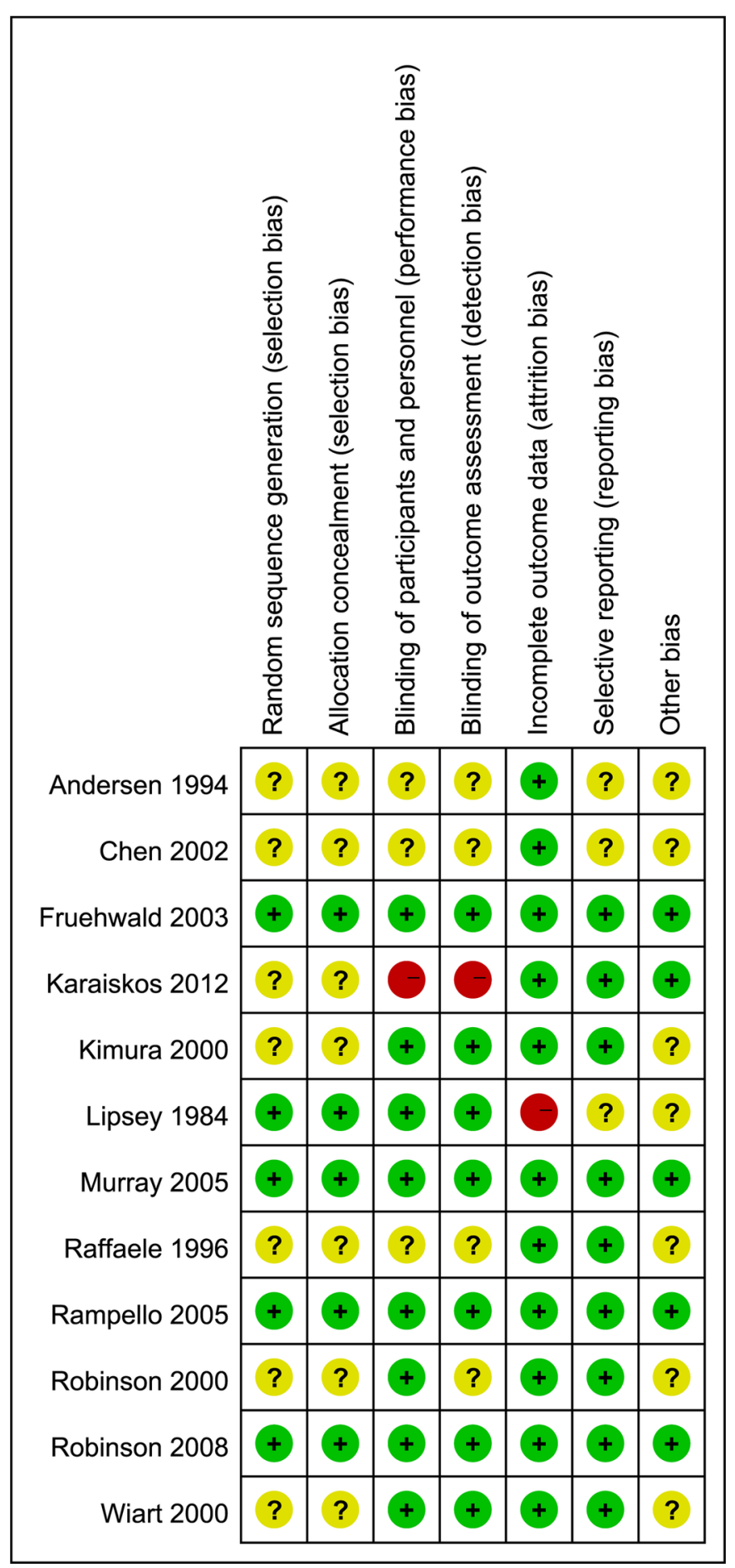

Figure 4 Risk of bias summary: review of the authors' judgements about each type of risk of bias for each included study.

terms were used, depending on the database. Studies were restricted to those published and unpublished in English, prior to November 2016, and the 'human' study. Two review authors independently decided on the selection based on title and abstract. Any disagreement between review authors was resolved by discussion. If there was still some disagreement, a further reviewer and expert (CZ) was consulted.
Outcome measures and data extraction

The primary outcomes were the overall efficacy. We defined overall efficacy as the mean change of the total score of the Hamilton Depression Rating Scale (HDRS) or Montgomery-Asberg Depression Rating Scale or Beck Depression Inventory or Bech-Rafaelsen Melancholia Scale or Zung Self-Rating Depression Scale (ZDS) from baseline to end point. When trials reported results from all of the above rating scales, we used them in the order described above. Intention-to-treat datasets were used whenever available.

The secondary outcome was the acceptability of the antidepressant treatment. We defined acceptability of treatment (treatment discontinuation) as the proportion of patients who left the study early for any reason out of the total number of patients randomly assigned to each antidepressant.

Because a multiple-treatments meta-analysis requires reasonable homogeneity, we focused on acute treatment, which we defined as 6 weeks' duration taking the main guidelines into account. ${ }^{91617}$ If 6-week data were not available, we used data from between 4 and 12 weeks (the data point closest to 6 weeks was given preference).

In the included studies, data were extracted by two reviewers (YS, HQ) independently. Once completed, any disagreements on data extraction and study evaluation were resolved through discussion. Information including study name, study characteristics, patient characteristics, depression diagnosis criteria, depression rating scale, comparators, dose range, treatment duration, sample size, effect sizes for two outcomes were extracted from each included study. Corresponding authors would be contacted for any missing information. If the article could not provide the mean change of the total score, we calculated the score based on a difference between the end point and the initial value or by measuring graphs presented in article.

We used network plots to explore the geometry of the treatment network of the included studies. The width of the lines is proportional to the number of studies comparing each pair of treatments, and the size of each node is proportional to the number of randomised participants (sample size).

The Cochrane risk of bias tool was used to assess the risk bias in the included studies. ${ }^{18}$ The tool contains seven domains, which are random sequence generation, allocation concealment, blinding of participants and personnel, blinding of outcome assessment, incomplete outcome data, selective reporting and other bias. The judgement for each domain includes a low risk of bias, a high risk of bias or unclear risk of bias. Two authors independently evaluated the risk of studies.

\section{Data synthesis and analysis}

We conducted two types of meta-analysis using a frequentist model. First, we conducted a pairwise meta-analysis for all direct comparisons with a random-effects model, ${ }^{19}$ assessing heterogeneity in these analyses with 
the $\mathrm{I}^{2}$ metric. ${ }^{20}$ Second, we fitted multiple-treatments meta-analysis models using the multivariate meta-regression approach proposed by White et $a l,{ }^{21-23}$ assuming a common heterogeneity variable for all comparisons (the tau $(\tau)$ value). $\tau$ is the estimated SD of underlying effects of treatment across studies in a meta-analysis. The relative effect sizes were calculated as standardised mean differences (Hedges' g) for continuous data (eg, the overall efficacy) or as ORs for binary outcomes (eg, the acceptability). Both types of effect sizes are reported with their 95\% CI.

Consistency assumption is the key to multiple-treatments meta-analysis, which implies that participants included in the network could hypothetically be randomised to any of the interventions represented in the network. ${ }^{24}$ For the assessment of consistency assumption, we initially conducted qualitative assessment based on clinical diversity, where we compared the distributions of characteristics that may modify the treatment effect. Subsequently, we evaluated the statistical disagreement of direct and indirect evidence (also known as inconsistency) in two ways. One was a loop-specific approach, ${ }^{25} 26$ in which inconsistent loops were identified as those yielding a 95\% CI excluding zero. The other was the design-by-treatment interaction model that provides a single inference, using the $\chi^{2}$ test, about the plausibility of assuming consistency throughout the entire network..$^{25}$ An inconsistency multiple-treatments

Treatment

Efficacy (SMD with 95\% CI)

Acceptability (OR with 95\% CI)

\begin{tabular}{|c|c|c|c|c|c|c|c|c|c|c|}
\hline REB & $\begin{array}{c}0.10 \\
(0.00,14.2)\end{array}$ & $\begin{array}{c}2.49 \\
(0.04,163.63)\end{array}$ & $\begin{array}{c}2.05 \\
(0.01,430.97)\end{array}$ & $\begin{array}{c}1.06 \\
(0.00,299.15)\end{array}$ & $\begin{array}{c}1.17 \\
(0.02,71.68)\end{array}$ & $\begin{array}{c}3.63 \\
(0.05,257.07)\end{array}$ & $\begin{array}{c}1.16 \\
(0.02,72.29)\end{array}$ & $\begin{array}{c}1.06 \\
(0.02,56.44)\end{array}$ & $\begin{array}{c}1.51 \\
(0.02,93.73)\end{array}$ & $\begin{array}{c}2.68 \\
(0.04,166.85)\end{array}$ \\
\hline$\frac{-3.84}{(-5.88,-1.80)}$ & PAR & $\frac{25.11}{(1.36,464.22)}$ & $\begin{array}{c}20.63 \\
(0.20,2171.47)\end{array}$ & $\begin{array}{c}10.69 \\
(0.07,1569.73)\end{array}$ & $\begin{array}{c}11,78 \\
(0.50,278.82)\end{array}$ & $\frac{36.55}{(1.28,1042.01)}$ & $\begin{array}{c}11.64 \\
(0.48,282.87)\end{array}$ & $\begin{array}{c}10.69 \\
(0.54,209.84)\end{array}$ & $\begin{array}{c}15.17 \\
(0.63,366.27)\end{array}$ & $\frac{26.98}{(1.12,652.09)}$ \\
\hline$\frac{-3.94}{(-5.97,-1.91)}$ & $\begin{array}{c}-0.10 \\
(-0.71,0.52)\end{array}$ & DOX & $\begin{array}{c}0.82 \\
(0.02,37.29)\end{array}$ & $\begin{array}{c}0.43 \\
(0.01,28.83)\end{array}$ & $\begin{array}{c}0.47 \\
(0.09,2.56)\end{array}$ & $\begin{array}{c}1.46 \\
(0.19,11.01)\end{array}$ & $\begin{array}{c}0.46 \\
(0.08,2.66)\end{array}$ & $\begin{array}{c}0.43 \\
(0.11,1.59)\end{array}$ & $\begin{array}{c}0.60 \\
(0.11,3.42)\end{array}$ & $\begin{array}{c}1.08 \\
(0.19,6.10)\end{array}$ \\
\hline$(-7.13,-2.96)$ & $(-2.37,-0.04)$ & $\begin{array}{c}-1.10 \\
(-2.26,0.05)\end{array}$ & DUL & $\begin{array}{c}0.52 \\
(0.00,111.25)\end{array}$ & $\begin{array}{c}0.57 \\
(0.01,23.89)\end{array}$ & $\begin{array}{c}1.77 \\
(0.05,61.67)\end{array}$ & $\begin{array}{c}0.57 \\
(0.02,19.63)\end{array}$ & $\begin{array}{c}0.52 \\
(0.01,18.52)\end{array}$ & $\begin{array}{c}0.74 \\
(0.02,31.28)\end{array}$ & $\begin{array}{c}1.31 \\
(0.03,55.70)\end{array}$ \\
\hline$(-7.22,-2.96)$ & $\frac{-1.25}{(-2.50,-0.00)}$ & $\begin{array}{c}-1.15 \\
(-2.40,0.09)\end{array}$ & $\begin{array}{c}-0.05 \\
(-1.37,1.27)\end{array}$ & TRA & $\begin{array}{c}1.10 \\
(0.02,68.89)\end{array}$ & $\begin{array}{c}3.42 \\
(0.05,246.89)\end{array}$ & $\begin{array}{c}1.09 \\
(0.02,69.47)\end{array}$ & $\begin{array}{c}1.00 \\
(0.02,54.29)\end{array}$ & $\begin{array}{c}1.42 \\
(0.02,90.08)\end{array}$ & $\begin{array}{c}2.52 \\
(0.04,160.35)\end{array}$ \\
\hline$\frac{-\frac{-5.31}{(-7.35,-3.28)}}{(-3)}$ & $\frac{-1.47}{(-2.55,-0.39)}$ & $\frac{-1.37}{(-2.45,-0.30)}$ & $\begin{array}{c}-0.27 \\
(-1.43,0.89)\end{array}$ & $\begin{array}{c}-0.22 \\
(-1.47,1.03)\end{array}$ & NOR & $\begin{array}{c}3.10 \\
(0.48,20.25)\end{array}$ & $\begin{array}{c}0.99 \\
(0.21,4.76)\end{array}$ & $\begin{array}{c}0.91 \\
(0.31,2.66)\end{array}$ & $\begin{array}{c}1.29 \\
(0.27,6.13)\end{array}$ & $\begin{array}{c}2.29 \\
(0.64,8.15)\end{array}$ \\
\hline$\frac{-\frac{-5.95}{(-7.91,-3.98)}}{2}$ & $(-3.04,-1.16)$ & $\frac{-2.01}{(-2.94,-1.07)}$ & $\frac{-0.90}{(-1.59,-0.22)}$ & $\begin{array}{c}-0.85 \\
(-1.98,0.28)\end{array}$ & $\begin{array}{c}-0.63 \\
(-1.57,0.30)\end{array}$ & CIT & $\begin{array}{c}0.32 \\
(0.05,1.95)\end{array}$ & $\begin{array}{c}0.29 \\
(0.06,1.36)\end{array}$ & $\begin{array}{c}0.42 \\
(0.06,2.80)\end{array}$ & $\begin{array}{c}0.74 \\
(0.11,4.99)\end{array}$ \\
\hline$\frac{-\frac{-5.93}{(-8.00,-3.85)}}{(8)}$ & $(-3.24,-0.94)$ & $\frac{-1.99}{(-3.14,-0.85)}$ & $(-1.57,-0.20)$ & $\begin{array}{c}-0.84 \\
(-2.15,0.47)\end{array}$ & $\begin{array}{c}-0.62 \\
(-1.77,0.53)\end{array}$ & $\begin{array}{c}0.02 \\
(-0.65,0.68)\end{array}$ & SER & $\begin{array}{c}0.92 \\
(0.29,2.89)\end{array}$ & $\begin{array}{c}1.30 \\
(0.26,6.53)\end{array}$ & $\begin{array}{c}2.32 \\
(0.46,11.63)\end{array}$ \\
\hline$\frac{-6.54}{(-8.42,-4.65)}$ & $\frac{-2.70}{(-3.46,1.93)}$ & $\frac{-2.60}{(-3.35,-1.84)}$ & $\frac{-1.49}{(-2.37,-0.62)}$ & 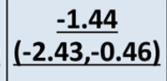 & $\frac{-1.22}{(-1.99,0.46)}$ & $(-1.14,-0.04)$ & $\begin{array}{c}-0.61 \\
(-1.47,0.25)\end{array}$ & PBA & $\begin{array}{c}1.42 \\
(0.46,4.40)\end{array}$ & $\begin{array}{c}2.52 \\
(0.81,7.84)\end{array}$ \\
\hline$\frac{-6.59}{(-8.53,-4.65)}$ & $(-3.63,-1.86)$ & $(-3.52,-1.77)$ & $\frac{-1.54}{(-2.53,-0.56)}$ & $\frac{-1.50}{(-2.58,-0.41)}$ & $\frac{-1.28}{(-2.16,-0.39)}$ & $\begin{array}{c}-0.64 \\
(-1.35,0.06)\end{array}$ & $\frac{-0.87}{(-1.49,-0.25)}$ & $\begin{array}{c}-0.51 \\
(-1.19,0.17)\end{array}$ & NEF & $\begin{array}{c}1.78 \\
(0.36,8.82)\end{array}$ \\
\hline$(-9.05,-5.04)$ & $(-4.22,-2.18)$ & $(-4.12,-2.09)$ & $\left(-3 . \frac{-2.00}{11,-0.89)}\right.$ & $\left(-3 . \frac{-1.95}{15,-0.75)}\right.$ & $(-2.49,-0.97)$ & $\left(-1 . \frac{-1.10}{97,-0.23)}\right.$ & $\frac{-1.33}{(-2.14,-0.52)}$ & $\begin{array}{c}0.51 \\
(-1.18,0.17)\end{array}$ & $\begin{array}{c}-0.46 \\
(-1.27,0.35)\end{array}$ & FLU \\
\hline
\end{tabular}

Figure 5 Efficacy and acceptability of the 11 antidepressants for PSD. Drugs are reported according to efficacy ranking. Comparisons between treatments should be read from left to right and the estimate is in the cell in common between the column-defining treatment and the row-defining treatment. For efficacy, SMDs lower than 0 favour the column-defining treatment. For acceptability, ORs higher than 1 favour the column-defining treatment. To obtain SMDs for comparisons in the opposite direction, negative values should be converted into positive values and vice versa. To obtain ORs for comparisons in the opposite direction, reciprocals should be taken. Significant results are in bold and underlined. CIT, citalopram; DOX, doxepin; DUL, duloxetine; FLU, fluoxetine; NEF, nefiracetam; NOR, nortriptyline; PAR, paroxetine; PBA, placebo; PSD, poststroke depression; REB, reboxetine; SER, sertraline; SMD, standard mean differences; TRA, trazodone. 


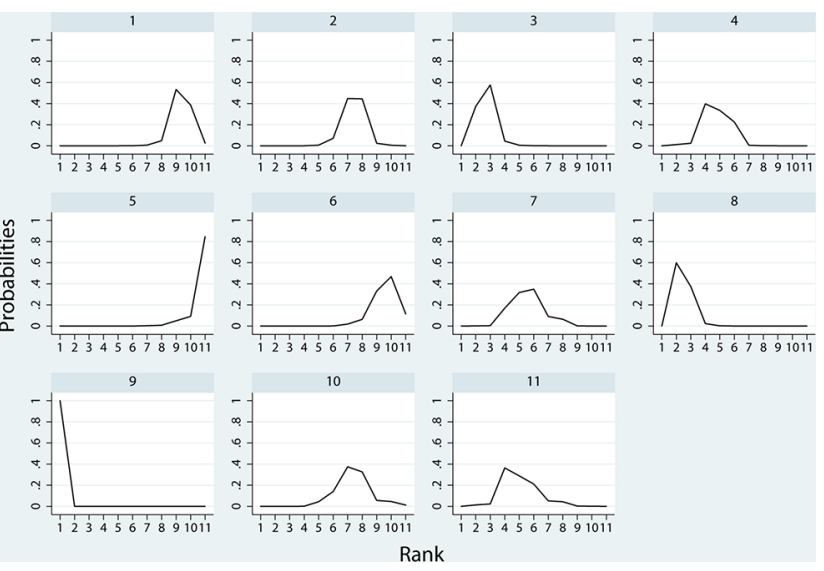

Figure 6 Ranking for efficacy. Ranking indicates the probability to be the best treatment, the second best, the third best and so on, among the 11 antidepressants: (1) placebo; (2) citalopram; (3) doxepin; (4) duloxetine; (5) fluoxetine; (6) nefiracetam; (7) nortriptyline; (8) paroxetine; (9) reboxetine; (10) sertraline; (11) trazodone.

meta-analysis model would be fitted when statistical inconsistency was present.

To rank the treatments for an outcome, we used surface under the cumulative ranking (SUCRA) probabilities, which are expressed as a percentage the total efficacy or acceptability of every intervention relative to an imaginary intervention that is always the best without uncertainty. ${ }^{23}$ Thus, large SUCRA scores should indicate a more effective or acceptable intervention.

The funnel plot was used to identify the possible publication bias if the number of studies was larger than 10 . We conducted several sensitivity analyses on the primary outcome to explore potential reasons for heterogeneity or inconsistency, such as whether it was early treatment. It has to be recalled that in the early stage (during the first 3 to 4 months after a stroke), PSD poses serious problems, such as worsened functional ${ }^{27} 28$ and vital prognoses ${ }^{27} 29$

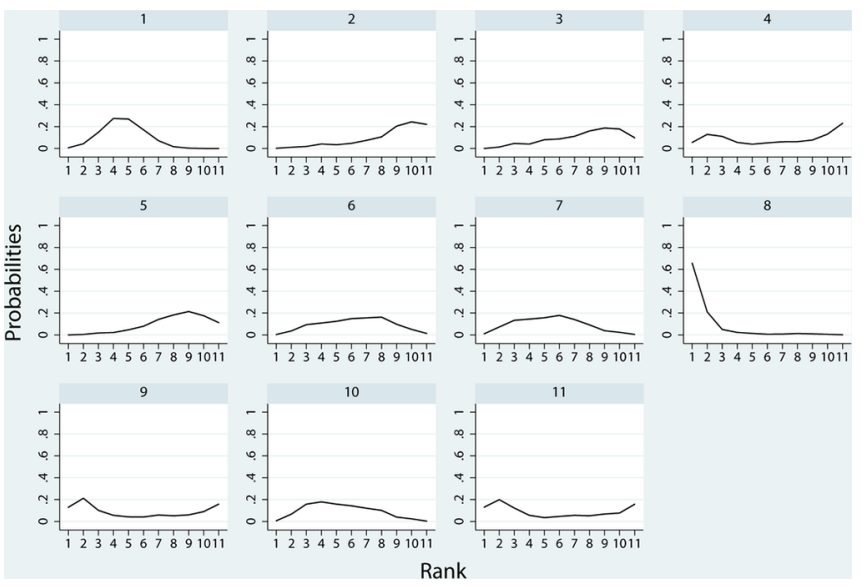

Figure 7 Ranking for acceptability. Ranking indicates the probability to be the best treatment, the second best, the third best and so on, among the 11 antidepressants: (1) placebo; (2) citalopram; (3) doxepin; (4) duloxetine; (5) fluoxetine; (6) nefiracetam; (7) nortriptyline; (8) paroxetine; (9) reboxetine; (10) sertraline; (11) trazodone.

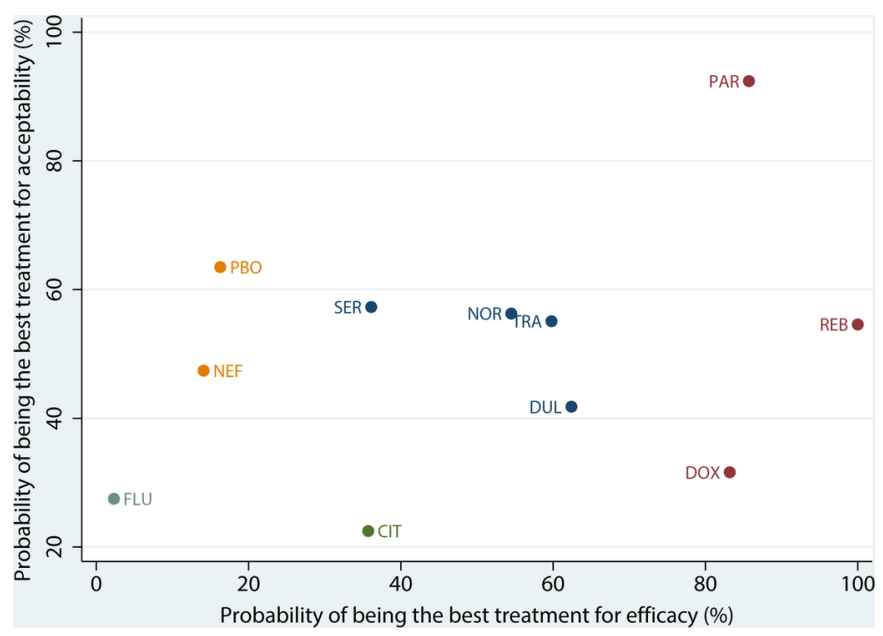

Figure 8 Clustered ranking plot for efficacy and acceptability. CIT, citalopram; DOX, doxepin; DUL, duloxetine; FLU, fluoxetine; NEF, nefiracetam; NOR, nortriptyline; PAR, paroxetine; PBA, placebo; REB, reboxetine; SER, sertraline; TRA, trazodone.

as well as worsened quality of life of the patient and his/ her caregiver, ${ }^{30}$ and therefore we defined the start of antidepressant treatment within 3 months after a stroke as early treatment. Meta-analyses were performed with Stata V.13.1 (StataCorp), using a suite of "network ${ }^{, 23}$ and mvmeta command. ${ }^{22}$

\section{RESULTS}

\section{Study identification and characteristics}

The Preferred Reporting Items for Systematic Reviews and Meta-Analyses flow diagram of studies selection was depicted in figure 1. A total of 9651 references in the primary search were identified. After removal of the duplicates, 4026 records were screened. Of these, 3989 were excluded based on screening of titles and abstracts

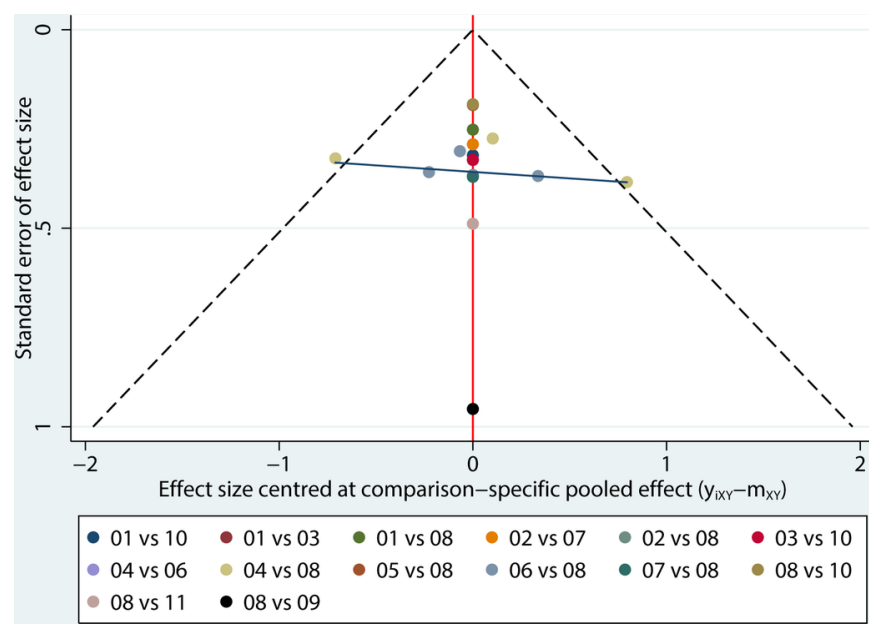

Figure 9 Comparison-adjusted funnel plot for efficacy. (01) citalopram; (02) doxepin; (03) duloxetine; (04) fluoxetine; (05) nefiracetam; (06) nortriptyline; (07) paroxetine; (08) placebo; (09) reboxetine; (10) sertraline; (11) trazodone. 


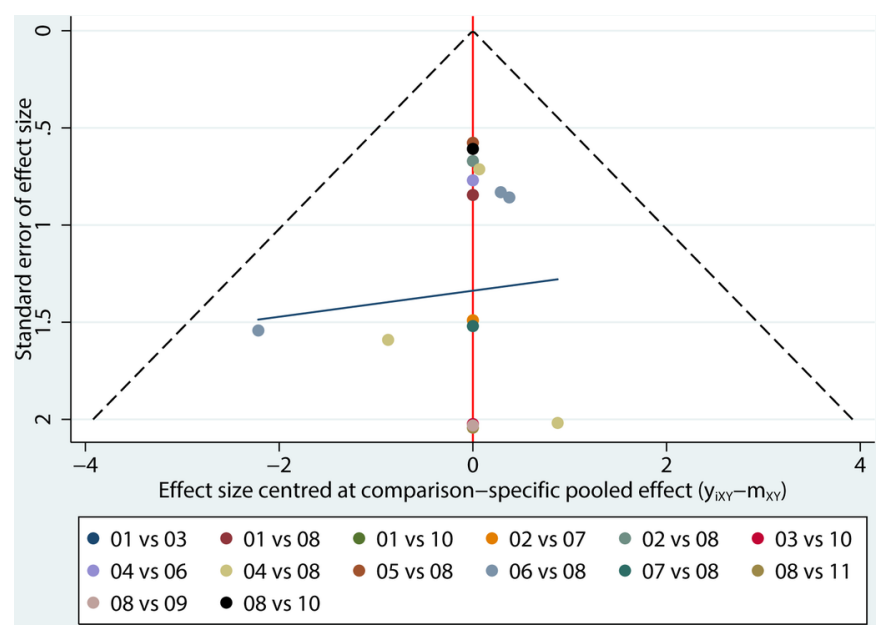

Figure 10 Comparison-adjusted funnel plot for acceptability. (01) citalopram; (02) doxepin; (03) duloxetine; (04) fluoxetine; (05) nefiracetam; (06) nortriptyline; (07) paroxetine; (08) placebo; (09) reboxetine; (10) sertraline; (11) trazodone.

and thus 37 were included in the narrative review, and data from 12 of these studies ${ }^{31-42}$ were included in the meta-analysis. In the study conducted by Robinson et $a l,{ }^{41}$ we selected the comparison between nefiracetam $(600 \mathrm{mg})$ and placebo instead of the comparison between nefiracetam $(900 \mathrm{mg})$ and placebo, because there was no statistically significant difference between the two doses. Whereas, the group of patients receiving nefiracetam $(600 \mathrm{mg})$ seemed to enjoy greater efficacy when the treatment duration was 9 weeks.

The characteristics of 12 studies published from 1984 to 2012 are shown in online supplementary file 2. A total of 707 participants were included in the review and the study sample size ranged from 22 to 123 . The mean age of all patients was above 50 . About half of the participants $(51 \%)$ were female and $42 \%$ had a right-sided stroke location. With respect to the nine studies ${ }^{31} 3335-4042$ in which a specific time of assessment after stroke was available, three studies ${ }^{32} 341$ were early treatment. Treatment duration ranged from 4 weeks to 12 weeks.

The network plot of the included studies for the multiple-treatments meta-analysis for efficacy is shown in figure 2. A total of 11 antidepressants were eligible, including placebo. Eighteen possible comparisons could be made, 12 of which were examined directly in 1 study,and two of which (nortriptyline vs placebo, fluoxetine vs placebo) were examined directly in three studies. The network plot of the included studies for acceptability analysis was similar.

\section{Risk of bias in included studies}

Figure 3 and 4 reveal the risk bias in all 12 studies. Five studies described random sequence generation and adequate allocation concealment. ${ }^{33} 36373941$ Eight studies described blinding of participants and personnel, ${ }^{33}$ 35-37 39-42 while one study had a high risk of bias since it was an open label. ${ }^{34}$ The study, an openlabel trial, was also considered as a high risk of bias about blinding of outcome assessment, ${ }^{34}$ while eight studies exhibited a low risk. ${ }^{33}$ 35-37 394142 All of studies had a low risk of incomplete outcome data ${ }^{31-35}$ 37-42 except for one. ${ }^{36}$ Seven studies had a low risk of selectively reporting results. ${ }^{32} 343638394142$

\section{Efficacy analysis and acceptability analysis}

The results of the direct comparisons for efficacy and acceptability analysis are shown in the online supplementary file 3 .

Figure 5 summarises the results of the multiple-treatments meta-analysis. With respect to overall efficacy, all drugs were significantly superior to placebo (range of mean effect sizes -0.59 to -6.54 ) apart from sertraline, nefiracetam and fluoxetine. Reboxetine was significantly more effective than all of the other drugs. With respect to acceptability (all-cause discontinuation), most of the comparisons revealed no significant differences except that paroxetine had significantly lower all-cause discontinuation than doxepin, citalopram and fluoxetine.

Figure 6 and 7 show the distribution of probabilities of 11 treatments being ranked for efficacy and acceptability, respectively. For the efficacy rank (figure 6), reboxetine, paroxetine, doxepin, duloxetine were among the most efficacious treatments, the cumulative probabilities of which were $100 \%, 85.7 \%, 83.2 \%, 62.4 \%$, respectively. The following drugs were less effective; trazodone (59.8\%), nortriptyline (54.5\%), citalopram (35.7\%), sertraline $(36.1 \%)$, placebo $(16.3 \%)$, nefiracetam $(14.1 \%)$, fluoxetine $(2.3 \%)$. With respect to the acceptability rank (figure 7), paroxetine, placebo, sertraline and nortriptyline were among the most acceptable treatments, the cumulative probabilities of which were $92.4 \%$, $63.5 \%, 57.3 \%$ and $56.3 \%$. The following drugs were less acceptable; trazodone $(55.1 \%)$, reboxetine (54.6\%), nefiracetam $(47.4 \%)$, duloxetine $(41.8 \%)$, doxepin $(31.6 \%)$, fluoxetine $(27.5 \%)$ and citalopram (22.5\%). Figure 8 shows the clustered ranking based on the probabilities of efficacy and acceptability. The exploration of inconsistency is described in detail in the online supplementary file 4 .

\section{Reporting biases and sensitivity analyses}

Comparison-adjusted funnel plots for efficacy (figure 9) and acceptability (figure 10) reveal the reporting bias in all 12 studies. Both of the plots were basically symmetrical.

Sensitivity analyses where the three studies with the early treatment with antidepressants for PSD (online supplementary file 5) were excluded did not substantially change the results for efficacy and acceptability, excepting that citalopram and sertraline exchanged the ranking order in SUCRA rank for efficacy, and sertraline and nortriptyline exchanged the ranking order in SUCRA rank for acceptability. In other words, sertraline became more effective but less acceptable after removal of the three studies with the early treatments. 


\section{DISCUSSION}

Although PSD is one of the most common complications after stroke and has been recognised by psychiatrists for more than 100 years, controlled systematic studies did not begin until the 1970s. ${ }^{43}$ However, there is still no consensus on the efficacy of antidepressants to treat PSD, and the relevant RCTs are few. At present, the Guidelines for Adult Stroke Rehabilitation and Recovery: A Guideline for Healthcare Professionals from the American Heart Association/American Stroke Association only indicated that treatment with heterocyclic antidepressant medications (tricyclic and tetracyclic antidepressants (TCAs)) and selective serotonin reuptake inhibitors (SSRIs) were stated as viable options for PSD. ${ }^{13}$ However, we found that not every TCA or SSRI is viable. In our study, reboxetine, a novel selective norepinephrine reuptake inhibitor (NARI) was ranked first for efficacy followed by paroxetine (SSRI), doxepin (TCA), duloxetine (serotonin and norepinephrine reuptake inhibitors (SNRI)), trazodone (serotonin receptor antagonists and serotonin reuptake inhibitors), nortriptyline (TCA), citalopram (SSRI), sertraline (SSRI), placebo, nefiracetam (nicotinic acetylcholine receptors $(\mathrm{nAChR}))$, fluoxetine (SSRI). In terms of acceptability, paroxetine (SSRI), placebo, sertraline (SSRI), nortriptyline (TCA) and reboxetine (NARI) were better tolerated than trazodone (serotonin receptor antagonists and serotonin reuptake inhibitors), nefiracetam (nAChR), duloxetine (SNRI), doxepin (TCA), fluoxetine (SSRI) and citalopram (SSRI). When weighing the efficacy and acceptability, it seems that paroxetine might be the best choice when starting an acute treatment for PSD, and fluoxetine might be the worst choice. Here, acute treatment referred to the treatment duration of 6-12 weeks. There are several studies suggesting that the results of comparison between different drugs would change as the treatment duration became longer, ${ }^{33} 34364041$ which indicated that the results of the ranking may also change. Clinicians need to know whether (and to what extent) antidepressant drug treatments work within a clinically reasonable period.

However, the results of the ranking are only for reference and are subject to the following limitations. First, although the examined related RCTs were heterogeneous, typically they had small sample sizes. ${ }^{1}$ Due to the variety of antidepressants, the research objectives were somewhat fragmented, and the number of studies on the same kind of antidepressant was too small, which meant that most of the comparisons in this review included only one study. Second, the results of inconsistency test for efficacy when examined by the loop-specific approach indicated that the loop of fluoxetine versus nortriptyline versus placebo was heterogeneous, and the inconsistency factor (IF) was obvious (IF=1.23) even though the loop was consistent. So we should cautiously explain the result of the loop. In addition, we did not take the subtype of PSD into account, because the inconsistency test revealed no significant difference even after excluding those studies which identified the subtypes of the participants. ${ }^{37}{ }^{39}$ It should be noted that the response of the patients with PSD might be related to the subtype of depression, similarly to what was observed for primary depression. ${ }^{44}{ }^{45}$ Therefore, it is important to emphasise that in this review, reboxetine was administered to patients with 'retarded' PSD. ${ }^{39}$ With respect to sertraline, the indirect comparison was consistent with the direct comparison, although the direct comparison of sertraline versus placebo had been undertaken in patients with stroke and minor depression and less severe major depression. ${ }^{37}$ For this reason, we speculated that the result of sertraline may not be related to the severity of depression. However, sertraline became more effective but less acceptable after removal of the three studies with the early treatments. We cannot judge whether the outcomes of early treatment is related to the severity of depression. Furthermore, some studies have shown that PSD was related with other factors such as sex, ${ }^{46}$ stroke location, ${ }^{47}$ which may influence the results. But it was not possible to carry out the relevant analyses due to the lack of individual data. Finally, we did not investigate important outcomes of acceptability, such as side effects, toxic effects and discontinuation symptoms, as only a few studies reported this kind of data. Therefore, the treatment discontinuation may not have been attributable only to the antidepressant itself.

Conventional pairwise meta-analyses of the treatment of antidepressants in PSD were less, ${ }^{78-52}$ few of which conducted meta-analysis based on different antidepressants. Tan $e t a l^{48}$ showed that citalopram was superior to the other SSRIs and TCAs in improving the total HDRS scores of PSD with acute treatment. The only other SSRIs included in that study were fluoxetine and sertraline. Nonetheless, the results were consistent with our rank order. The TCAs included also amitriptyline and imipramine, which were not included in our analysis since we excluded Chinese articles. $\mathrm{Xu}$ et $a l^{7}$ demonstrated a significant advantage associated with antidepressants in comparison with placebo treatment in PSD $(\mathrm{SMD}=-0.96$; $95 \%$ CI -1.41 to $-0.51 ; \mathrm{p}<0.0001)$. In that previous meta-analysis, the subgroup analyses demonstrated that when compared with placebo, other antidepressants $(\mathrm{SMD}=-2.01 ; 95 \% \mathrm{CI}-3.13$ to $-0.89 ; \mathrm{p}=0.10)$ were better than TCAs (SMD $=-1.41 ; 95 \%$ CI -2.51 to -0.31 ; $\mathrm{p}=0.02)$ and SSRIs (SMD $=-0.53 ; 95 \%$ CI -0.97 to -0.09 ; $\mathrm{p}<0.0001$ ), which are not at odds with the results found here.

All in all, in the treatment of PSD, there are many unresolved factors that could influence the choice of the optimal antidepressant compound that is, subtype of depression, sex, stroke location. One topic for future exploration would be to determine whether the duration of treatment affects the response. There is an urgent 
need for more high-quality research on antidepressant treatment in PSD.

Contributors YS designed the study, extracted the data, wrote and approved the manuscript. YL ran the network meta-analysis, revised and approved the manuscript. YJ and JL identified the relevant outcomes, discussed the validity of the trials, revised and approved the manuscript. $\mathrm{HQ}$ extracted the data, proofread the results and approved the manuscript. JX reanalysed data to match inclusion/exclusion criteria, revised and approved the manuscript. CZ conducted the systematic review, evaluated the quality of the studies, participated in the elaboration of the manuscript and approved the manuscript.

Funding This work was supported by The Program of Liaoning Province Education Administration (no LK201605 to YS), The Shenyang Population and Health Technical Critical Special Project (no F16520659501 to CZ) and The Program of the Distinguished Professor of Liaoning Province, Neurology (to CZ).

Competing interests None declared.

Provenance and peer review Not commissioned; externally peer reviewed. Data sharing statement No additional data are available.

Open Access This is an Open Access article distributed in accordance with the Creative Commons Attribution Non Commercial (CC BY-NC 4.0) license, which permits others to distribute, remix, adapt, build upon this work non-commercially, and license their derivative works on different terms, provided the original work is properly cited and the use is non-commercial. See: http://creativecommons.org/ licenses/by-nc/4.0/

(c) Article author(s) (or their employer(s) unless otherwise stated in the text of the article) 2017. All rights reserved. No commercial use is permitted unless otherwise expressly granted.

\section{REFERENCES}

1. Towfighi A, Ovbiagele B, El Husseini N, et al. Poststroke Depression: A Scientific Statement for Healthcare Professionals From the American Heart Association/American Stroke Association. Stroke 2017;48.

2. Wulsin L, Alwell K, Moomaw CJ, et al. Comparison of two depression measures for predicting stroke outcomes. $J$ Psychosom Res 2012;72:175-9.

3. Willey JZ, Disla N, Moon YP, et al. Early depressed mood after stroke predicts long-term disability: the Northern Manhattan Stroke Study (NOMASS). Stroke 2010;41:1896-900.

4. Kim JH, Park EY. The factor structure of the center for epidemiologic studies depression scale in stroke patients. Top Stroke Rehabil 2012;19:54-62.

5. Jørgensen TS, Wium-Andersen IK, Wium-Andersen MK, et al. Incidence of Depression After Stroke, and Associated Risk Factors and Mortality Outcomes, in a Large Cohort of Danish Patients. JAMA Psychiatry 2016;73:1032-40.

6. Meader N, Moe-Byrne T, Llewellyn A, et al. Screening for poststroke major depression: a meta-analysis of diagnostic validity studies. $J$ Neurol Neurosurg Psychiatry 2014;85:198-206.

7. Xu XM, Zou DZ, Shen LY, et al. Efficacy and feasibility of antidepressant treatment in patients with post-stroke depression. Medicine 2016;95:e5349.

8. Hackett ML, Anderson CS, House A, et al. Interventions for preventing depression after stroke. Cochrane Database Syst Rev 2008:CD003689.

9. Holloway RG, Arnold RM, Creutzfeldt CJ, et al. Palliative and endof-life care in stroke: a statement for healthcare professionals from the American Heart Association/American Stroke Association. Stroke 2014;45:1887-916

10. Hackett ML, Yapa C, Parag V, et al. Frequency of depression after stroke: a systematic review of observational studies. Stroke 2005;36:1330-40.

11. Hackett ML, Pickles K. Part I: frequency of depression after stroke: an updated systematic review and meta-analysis of observational studies. Int J Stroke 2014;9:1017-25.

12. Hackett ML, Anderson CS, House A, et al. Interventions for treating depression after stroke. Cochrane Database Syst Rev 2008:CD003437.

13. Winstein CJ, Stein J, Arena R, et al. Guidelines for Adult Stroke Rehabilitation and Recovery: A Guideline for Healthcare Professionals From the American Heart Association/American Stroke Association. Stroke 2016;47:e98-e169.
14. Salanti G, Higgins JP, Ades AE, et al. Evaluation of networks of randomized trials. Stat Methods Med Res 2008:17:279-301.

15. Ades AE, Sculpher M, Sutton A, et al. Bayesian methods for evidence synthesis in cost-effectiveness analysis. Pharmacoeconomics 2006;24:1-19.

16. Eskes GA, Lanctôt KL, Herrmann N, et al. Canadian Stroke Best Practice Recommendations: Mood, Cognition and Fatigue Following Stroke practice guidelines, update 2015. Int J Stroke 2015;10:1130-40.

17. Intercollegiate Stroke Working Party. National clinical guideline for stroke. 4th edition: Royal College of Physicians, 2012.

18. Higgins J, Green S. Cochrane Handbook for Systematic Reviews of Interventions Version 5.1.0: The Cochrane Collaborationk, 2011. www.cochrane-handbook.org

19. DerSimonian R, Laird N. Meta-analysis in clinical trials. Control Clin Trials 1986;7:177-88.

20. Higgins JPT, Green S. Cochrane handbook for systematic reviews of interventions, version 5.1.0, 2011. http://www.cochrane-handbook. org (accessed 9 Apr 2015).

21. White IR. Multivariate random-effects meta-regression: updates to mvmeta. The STATA Journal 2011;11:255-70.

22. White IR, Barrett JK, Jackson D, et al. Consistency and inconsistency in network meta-analysis: model estimation using multivariate metaregression. Res Synth Methods 2012;3:111-25.

23. Chaimani A, Higgins JP, Mavridis D, et al. Graphical tools for network meta-analysis in STATA. PLoS One 2013;8:e76654.

24. Salanti G. Indirect and mixed-treatment comparison, network, or multiple-treatments meta-analysis: many names, many benefits, many concerns for the next generation evidence synthesis tool. Res Synth Methods 2012;3:80-97.

25. Veroniki AA, Vasiliadis HS, Higgins JP, et al. Evaluation of inconsistency in networks of interventions. Int $J$ Epidemiol 2013;42:332-45.

26. Salanti G, Marinho V, Higgins JP, et al. A case study of multipletreatments meta-analysis demonstrates that covariates should be considered. J Clin Epidemiol 2009;62:857-64.

27. Robinson RG, Morris PL, Fedoroff JP, et al. Depression and cerebrovascular disease. J Clin Psychiatry 1990;51:26-33.

28. Parikh RM, Robinson RG, Lipsey JR, et al. The impact of poststroke depression on recovery in activities of daily living over a 2-year follow-up. Arch Neurol 1990;47:785-9.

29. Morris PL, Robinson RG, Andrzejewski P, et al. Association of depression with 10-year poststroke mortality. Am J Psychiatry 1993;150:124-9.

30. Angeleri F, Angeleri VA, Foschi N, et al. The influence of depression, social activity, and family stress on functional outcome after stroke. Stroke 1993;24:1478-83.

31. Andersen G, Vestergaard K, Lauritzen L, et al. Effective treatment of poststroke depression with the selective serotonin reuptake inhibitor citalopram. Stroke 1994;25:1099-104.

32. Chen W, Wang G-F, Chen X-H, et al. Effects of paroxetine on function recovery in patients with poststroke depression. Chinese $\mathrm{J}$ Clin Rehabil 2002;6:2014-5.

33. Fruehwald S, Gatterbauer E, Rehak P, et al. Early fluoxetine treatment of post-stroke depression--a three-month double-blind placebocontrolled study with an open-label long-term follow up. J Neurol 2003;250:347-51.

34. Karaiskos D, Tzavellas E, Spengos K, et al. Duloxetine versus citalopram and sertraline in the treatment of poststroke depression, anxiety, and fatigue. J Neuropsychiatry Clin Neurosci 2012;24:349-53.

35. Kimura M, Robinson RG, Kosier JT, et al. Treatment of cognitive impairment after poststroke depression : a double-blind treatment trial. Stroke 2000;31:1482-6.

36. Lipsey JR, Robinson RG, Pearlson GD, et al. Nortriptyline treatment of post-stroke depression: a double-blind study. Lancet 1984;1:297-300

37. Murray V, von Arbin M, Bartfai A, et al. Double-blind comparison of sertraline and placebo in stroke patients with minor depression and less severe major depression. J Clin Psychiatry 2005;66:708-16.

38. Raffaele R, Rampello L, Vecchio I, et al. Trazodone therapy of the post-stroke depression. Arch Gerontol Geriatr 1996;22(Suppl 1):217-20.

39. Rampello L, Alvano A, Chiechio S, et al. An evaluation of efficacy and safety of reboxetine in elderly patients affected by "retarded" post-stroke depression. A random, placebo-controlled study. Arch Gerontol Geriatr 2005;40:275-85.

40. Robinson RG, Schultz SK, Castillo C, et al. Nortriptyline versus fluoxetine in the treatment of depression and in short-term recovery after stroke: a placebo-controlled, double-blind study. Am J Psychiatry 2000;157:351-9. 
41. Robinson RG, Jorge RE, Clarence-Smith K, et al. Double-blind randomized treatment of poststroke depression using nefiracetam. $J$ Neuropsychiatry Clin Neurosci 2008;20:178-84.

42. Wiart L, Petit $\mathrm{H}$, Joseph PA, et al. Fluoxetine in early poststroke depression: a double-blind placebo-controlled study. Stroke 2000;31:1829-32.

43. Robinson RG, Jorge RE. Post-Stroke Depression: a Review. Am J Psychiatry 2016;173:221-31.

44. Rampello L, Nicoletti G, Raffaele R, et al. Comparative effects of amitriptyline and amineptine in patients affected by anxious depression. Neuropsychobiology 1995;31:130-4.

45. Rampello L, Nicoletti G, Raffaele R, et al. Dopaminergic hypothesis for retarded depression: a symptom profile for predicting therapeutical responses. Acta Psychiatr Scand 1991;84:552-4.

46. Alajbegovic A, Djelilovic-Vranic J, Nakicevic A, et al. Post stroke depression. Med Arch 2014;68:47-50.
47. Wei N, Yong W, Li X, et al. Post-stroke depression and lesion location: a systematic review. J Neurol 2015;262:81-90.

48. Tan S, Huang X, Ding L, et al. Efficacy and safety of citalopram in treating post-stroke depression: a meta-analysis. Eur Neurol 2015;74:188-201.

49. Bhogal SK, Teasell R, Foley N, et al. Heterocyclics and selective serotonin reuptake inhibitors in the treatment and prevention of poststroke depression. J Am Geriatr Soc 2005;53:1051-7.

50. Hackett ML, Anderson CS, House AO, et al. Management of depression after stroke: a systematic review of pharmacological therapies. Stroke 2005;36:1092-7.

51. Chen Y, Guo JJ. Meta-analysis of antidepressant treatment for patients with poststroke depression. Stroke 2006;37:1365-6.

52. Chen Y, Guo JJ, Zhan S, et al. Treatment effects of antidepressants in patients with post-stroke depression: a meta-analysis. Ann Pharmacother 2006;40:2115-22. 